\title{
Predicting recurrent pulmonary embolism and chronic thromboembolic pulmonary hypertension: one more way to skin the cat
}

\author{
Frederikus A. Klok ${ }^{1}$ and Marius M. Hoeper ${ }^{2}$ \\ Affiliations: ${ }^{1}$ Dept of Medicine - Thrombosis and Hemostasis, Leiden University Medical Center, Leiden, The \\ Netherlands. ${ }^{2}$ Dept of Respiratory Medicine and German Center of Lung Research, Hannover Medical School, \\ Hannover, Germany.
}

Correspondence: F. A. Klok, Leiden University Medical Center, Dept of Thrombosis and Hemostasis, Room C7-14, Albinusdreef 2, 2300 RC Leiden, the Netherlands. E-mail: F.A.KlokaLUMC.nl

@ERSpublications

Persistent perfusion defects predict recurrent pulmonary embolism and CTEPH http://ow.ly/xMHm30aD7aB

Cite this article as: Klok FA, Hoeper MM. Predicting recurrent pulmonary embolism and chronic thromboembolic pulmonary hypertension: one more way to skin the cat. Eur Respir J 2017; 49: 1700413 [https://doi.org/10.1183/13993003.00413-2017].

In this issue of the European Respiratory Journal, PESAVENTO et al. [1] present the results of the Study on the Clinical Course Of Pulmonary Embolism (SCOPE). In this huge effort, 647 consecutive patients with a symptomatic, confirmed first episode of acute pulmonary embolism (PE) were recruited and subjected to lung scanning after an initial treatment period of 6 months to assess the presence of residual pulmonary obstruction. Patients were followed for an additional 3-year period to monitor the occurrence of recurrent venous thromboembolism (VTE) and/or chronic thromboembolic pulmonary hypertension (CTEPH). The primary aim of the SCOPE study was to evaluate the predictive value of persistent perfusion defects for recurrent $\mathrm{PE}$ and $\mathrm{CTEPH}$. Notably, $50 \%$ of patients were found to have some degree of persistent perfusion defects, with $56(8.7 \%)$ patients having a persistent perfusion obstruction of $>25 \%$ and none $>72 \%$. Both higher age (OR 1.03, 95\% CI 1.02-1.04, per year) and unprovoked PE (OR 1.40, 95\% CI 1.011.95) were predictive of persistent perfusion defects, whereas among others reperfusion therapy at baseline was not. During the 3-year follow-up period, 40 patients were diagnosed with recurrent VTE $(6.2 \%$; $95 \%$ CI 4.5-8.3) and 11 with CTEPH (1.7\%; 95\% CI 0.9-3.0). In multivariate COX regression analysis, persistent perfusion defects predicted the combined endpoint of recurrent VTE and/or CTEPH for an adjusted hazard ratio of 2.26 (95\% CI 1.23-4.16). From these results, the authors conclude that a single assessment of persistent perfusion defects at 6 months may guide treatment decisions regarding duration of anticoagulation therapy.

Despite the large sample size, blinded adjudication of the endpoints and robust statistical methods, several aspects of this study require consideration. First, although absolute risks of recurrent VTE and CTEPH in patients with and without persistent perfusion defects were presented, adjusted hazard ratios for recurrent PE and CTEPH separately were not provided. In addition, although not directly studied, the study results reinforce the notion that perfusion scanning has a higher sensitivity than computed tomography in detecting residual perfusion defects. Further, the risk of CTEPH and recurrent PE is very low in patients with provoked PE. Therefore, a separate analysis restricted to patients with unprovoked PE would have

Received: Feb 262017 | Accepted: Feb 282017

Conflict of interest: Disclosures can be found alongside this article at erj.ersjournals.com

Copyright OERS 2017 
facilitated better translation of the study results to clinical practice. Finally, and importantly, the treating physicians were aware of the result of the lung scan, which may have influenced therapeutic management. Indeed, patients with persistent perfusion defects were significantly more likely to be treated with anticoagulants beyond the first 6 months. This important bias however is indicative of an even better predictive value of persistent perfusion defects for recurrent VTE than found in this study.

Should we now perform reference perfusion scans in all our PE patients to guide treatment duration and intensity of CTEPH-specific follow-up? The answer to that question has to be "no". This was not a randomised controlled outcome study. Therefore, the results of the SCOPE study are hypothesis generating rather than practice changing. Moreover, lung scanning has to compete with a whole range of predictive tests for recurrent VTE, including D-dimer testing and other global coagulation assays, as well as several multivariate VTE risk assessment models [2-6]. Notably, none of these tests has been unequivocally proven to improve the patients' prognosis when applied as primary tool to guide treatment decisions, nor are they recommend by recent guidelines [7]. Of note, the availability of imaging tests performed after discontinuation of anticoagulant treatment has been suggested to provide a useful basis for comparisons in cases of suspected recurrent PE [8-10]. The authors however do not provide details on this subject since it was not the primary aim of their study. Generally, and in contrast to deep vein thrombosis (DVT) of the legs, where a reference compression ultrasound is often crucial for the correct diagnosis of recurrent DVT, reference imaging after acute PE has not been demonstrated to be useful for the management of suspected recurrent PE [9-11].

As for the prediction of CTEPH, up till now, no screening test has been shown to be associated with a faster CTEPH diagnosis or improved outcome. With 50\% abnormal lung scans and only $3.4 \%$ of patients with an abnormal scan developing CTEPH, both the low frequency of CTEPH and the specificity of lung scanning for CTEPH do not justify the costs and the radiation exposure associated with performing this test in all patients with (unprovoked) PE $[12,13]$.

Although this study does not change our current practice, the results of this study are nevertheless important and provide unique new insight into the pathophysiology of CTEPH after PE. Specifically, since none of the patients with normalised lung scans developed CTEPH, incomplete thrombus resolution despite anticoagulant treatment is very likely to be the key trigger for the development of CTEPH. We should also bear in mind that, especially in patients with unprovoked PE, the first clinically apparent episode of PE may have been preceded by unrecognised asymptomatic events, which may explain the high rate of residual perfusion defects in these patients. Similar lines of thought have been applied to explain the high variability in the reported incidence of $\mathrm{CTEPH}$ in survivors of acute $\mathrm{PE}$, which ranges from $0.1 \%$ to more than $10 \%[13,14]$. It is likely that many of these patients had already developed CTEPH after repeated asymptomatic embolic events before presenting with the clinical picture of acute PE.

Future studies aiming at unravelling the aetiology of CTEPH should focus on the mechanisms underlying impaired endogenous thrombolysis. Recent studies have shed more light on processes that impair thrombus resolution and identified impaired angiogenesis, inflammation as well as hypoxia-induced activation of endothelial, mesenchymal and immune cells to be key suspects to inhibit physiological thrombus resolution, contributing to the development of CTEPH $[15,16]$.

\section{References}

1 Pesavento R, Filippi L, Palla A, et al. Impact of residual pulmonary obstruction on the long-term outcome of patients with pulmonary embolism. Eur Respir J 2017; 49: 1601980.

2 Christiansen SC, Cannegieter SC, Koster T, et al. Thrombophilia, clinical factors, and recurrent venous thrombotic events. JAMA 2005; 293: 2352-2361.

3 Palareti G, Cosmi B, Antonucci E, et al. Duration of anticoagulation after isolated pulmonary embolism. Eur Respir J 2016; 47: 1429-1435.

4 Rodger MA, Kahn SR, Wells PS, et al. Identifying unprovoked thromboembolism patients at low risk for recurrence who can discontinue anticoagulant therapy. CMAJ 2008; 179: 417-426.

5 Eichinger S, Heinze G, Jandeck LM, et al. Risk assessment of recurrence in patients with unprovoked deep vein thrombosis or pulmonary embolism: the Vienna prediction model. Circulation 2010; 121: 1630-1636.

6 Tosetto A, Iorio A, Marcucci M, et al. Predicting disease recurrence in patients with previous unprovoked venous thromboembolism: a proposed prediction score (DASH). J Thromb Haemost 2012; 10: 1019-1025.

7 Konstantinides SV, Torbicki A, Agnelli G, et al. 2014 ESC guidelines on the diagnosis and management of acute pulmonary embolism. Eur Heart J 2014; 35: 3033-3069.

8 Hamadah A, Alwasaidi T, Le GG, et al. Baseline imaging after therapy for unprovoked venous thromboembolism: a randomized controlled comparison of baseline imaging for diagnosis of suspected recurrence. J Thromb Haemost 2011; 9: 2406-2410.

9 Le Gal G, Kovacs MJ, Carrier M, et al. Validation of a diagnostic approach to exclude recurrent venous thromboembolism. J Thromb Haemost 2009; 7: 752-759.

10 Huisman MV, Klok FA. Diagnostic management of acute deep vein thrombosis and pulmonary embolism. J Thromb Haemost 2013; 11: 412-422.

11 Prandoni P, Lensing AW, Bernardi E, et al. The diagnostic value of compression ultrasonography in patients with suspected recurrent deep vein thrombosis. Thromb Haemost 2002; 88: 402-406. 
12 Ende-Verhaar YM, Huisman MV, Klok FA. To screen or not to screen for chronic thromboembolic pulmonary hypertension after acute pulmonary embolism. Thromb Res 2017; 151: 1-7.

13 Ende-Verhaar YM, Cannegieter SC, Vonk Noordegraaf A, et al. Incidence of chronic thromboembolic pulmonary hypertension after acute pulmonary embolism: a contemporary view of the published literature. Eur Respir J 2017; 49: 1601792.

14 Simonneau G, Hoeper MM. Evaluation of the incidence of rare diseases: difficulties and uncertainties, the example of chronic thromboembolic pulmonary hypertension. Eur Respir J 2017; 49: 1602522.

15 Bochenek ML, Rosinus NS, Lankeit M, et al. From thrombosis to fibrosis in chronic thromboembolic pulmonary hypertension. Thromb Haemost 2017; 117: 769-783.

16 Quarck R, Wynants $\mathrm{M}$, Verbeken E, et al. Contribution of inflammation and impaired angiogenesis to the pathobiology of chronic thromboembolic pulmonary hypertension. Eur Respir J 2015; 46: 431-443. 